\title{
Novel Population Pharmacokinetic Method Compared to the Standard Noncompartmental Approach to Assess Bioequivalence of Iron Gluconate Formulations
}

\author{
Corinne Seng Yue ${ }^{1-2}$, Keith Gallicano ${ }^{3}$, Line Labbé ${ }^{2}$, Murray P Ducharme ${ }^{1-2}$ \\ ${ }^{1}$ Learn and Confirm Inc., 3630 Bois Franc, St-Laurent QC, Canada. ${ }^{2}$ Université de Montréal, Faculté de pharmacie, Pavillon \\ Jean Coutu, 2940 Chemin de la polytechnique, Montreal QC, Canada. ${ }^{3}$ Watson Laboratories Inc., Corona, USA.
}

Received April 9, 2013; Accepted, June 21, 2013; Published, June 23, 2013.

\begin{abstract}
Purpose: Iron-containing products are atypical in terms of their pharmacokinetic properties because iron is only removed by plasma sampling and is non-linear. This study aims to present a novel way of assessing the relative bioavailability of two sodium ferric gluconate complex (SFGC) formulations and compare this approach to a standard previously published noncompartmental approach. Methods: Data were from openlabel, randomized, single-dose studies (Study 1 was parallel whereas Study 2 was crossover). Subjects with low but normal iron levels were infused IV SFGC in sucrose by GeneraMedix Inc. and/or Ferrlecit ${ }^{\circledR}$ Injection (Watson Laboratories Inc.). In Study 1 ( $\mathrm{n}=240), 125 \mathrm{mg}$ was infused over 10 minutes. In Study 2 ( $\mathrm{n}=29), 62.5$ mg was infused over 30 minutes. Samples were assayed for total iron (TI) and transferrin-bound iron (TBI) over 36 hours (Study 1) or 72 hours (Study 2) post-dose. Studies 1 and 2 used standard noncompartmental analysis. Study 2 also used population PK (PPK) analyses with ADAPT $5 \AA$. The final model predicted SFGC area-underthe-curve $\left(\mathrm{AUC}_{\mathrm{pred}}\right)$ and maximal concentration $\left(\mathrm{Cmax}_{\mathrm{pred}}\right)$. Analyses of variance was conducted on $\mathrm{ln}$ transformed PK parameters. Ratios of means and 90\% confidence intervals (CIs) were estimated. Bioequivalence was demonstrated if values were within 80-125\%. Results: For Study 1, ratios and 90\% CIs for TI baseline-corrected $\mathrm{Cmax}$ and $\mathrm{AUC}_{0-36}$ were 100.4 (96.5 - 104.5) and 99.7 (94.2 - 105.5). For TBI, results for TI baseline-corrected Cmax and $\mathrm{AUC}_{0-36}$ were 86.8 (82.7 - 91.1) and 92.4 (85.6 - 99.7). For Study 2, a multicompartmental model simultaneously described the PK of TI, TBI and SFGC. Ratios and 90\% CIs for SFGC $\mathrm{Cmax}_{\text {pred }}$ and $\mathrm{AUC}_{\text {pred }}$ were 89.9 (85.9 - 94.0) and 89.7 (85.7 - 93.9), while ratios and 90\% CI obtained from the noncompartmental analysis of Study 2 did not meet BE criteria because of low power. Conclusions: Both the standard and PPK modeling approach suggested bioequivalence between the iron products. However, with the PPK method, less subjects were required to meet study objectives compared to the standard noncompartmental approach which required considerably more subjects (29 vs 240 ).
\end{abstract}

This article is open to POST-PUBLICATION REVIEW. Registered readers (see "For Readers") may comment by clicking on ABSTRACT on the issue's contents page.

\section{INTRODUCTION}

Iron plays several important roles in the human body, by participating in transmembrane transport, electron transfers, DNA synthesis and acting as a co-factor in enzymatic reactions (especially those involving heme) $(1,2)$. In addition to these functions, iron plays a vital part in the transport of oxygen via the heme molecule, a porphyrin ring structure with a central iron atom $(1,2)$. It is also a component of enzymes such as peroxidase, myeloperoxidase, amino acid hydroxylase and 5lipoxygenase (2).
Because of its vital importance to so many bodily functions, severe iron deficiency is often treated with intravenous administration of iron. Parenteral iron was first administered over a century ago, and since then various intravenous formulations of iron have been developed (3). Although the different iron nanoparticles (iron dextran, iron sucrose, sodium ferric gluconate,

\footnotetext{
Corresponding Author: Murray P Ducharme; 3630 Bois Franc; St-Laurent, QC; Canada; Email: murray.ducharme@learnandconfirm.ca
} 
ferumoxytol) exhibit various characteristics, once the iron is internalized, it is taken up by the reticuloendothelial system (RES) composed of monocytes and macrophages in the liver, spleen and bone marrow $(2,4,5)$. Subsequently, it is bound to transferrin, either intracellularly in pools or extracellularly for transport to erythrocytes. It should be noted that a small fraction of iron likely binds to extracellular transferrin directly from the plasma (4). Only a small amount of iron is excreted daily in the urine and feces (around $1 \mathrm{mg}$ or $0.03 \%$ of the average body's total stores), as there is no true excretion pathway for iron, and the loss from desquamation of skin cells and sweat is negligible $(1,5,6,7)$. Thus, iron requirements for erythropoiesis are generally met through the recycling of iron from senescent erythrocytes $(1,5)$.

The pharmacokinetics (PK) of iron appears to be non-linear, as demonstrated by the saturable plasma clearance of iron dextran, which appears to reach a plateau after doses exceeding $500 \mathrm{mg}$ (8). This non-linearity is thought to occur between the distribution of iron taken up by the RES that subsequently binds to transferrin. In addition, it is virtually not eliminated from the body, as it is only lost through phenomena such as blood donations/sampling or through blood loss and hemorrhagic events $(2,9)$. Because of these particular PK characteristics, iron does not lend itself well to noncompartmental PK analyses (10). Indeed, it violates certain basic assumptions of noncompartmental analysis such as linearity and constant elimination from the sampling compartment $(10,11,12)$. In addition, the endogenous baseline concentration of iron is not constant and changes significantly after iron dosing simply because iron is not eliminated. All of this can pose problems when using the noncompartmental approach to derive baseline adjusted parameters such as the maximal observed serum or plasma concentration (Cmax) and the area under the plasma/serum concentration time curve (AUC) as this method assumes linearity in the PK of iron and in its baseline levels. These PK parameters are often used to assess the relative bioavailability (BA) between two formulations, a process that compares "the rate and extent to which the active drug ingredient or therapeutic moiety is absorbed from a drug product and becomes available at the site of drug action" (13). This comparison is central to the bioequivalence (BE) assessment, which aims at determining if administration of the same molar dose of the same active ingredient or moiety under identical conditions leads to similar BA, or BA that is not significantly different.

Specific BE guidelines outlining the ideal study design and statistical approaches that are preferred have been published by various regulatory (government) agencies $(14,15,16,17)$, and for most drug products, $\mathrm{BE}$ is generally assessed by comparing the average BA parameters (Cmax and AUC) of two products. Study designs tend to be two-period, two-treatment, two-sequence crossovers conducted in healthy volunteers, to minimize variability by administering the test and reference products to the same subjects. Using the $1 n-$ transformed PK parameters calculated for each subject, analyses of variance (ANOVA) are then performed and least square mean (LSM) ratios of test to reference PK parameters and $90 \%$ confidence intervals (CIs) associated with the ratios are obtained. Pre-defined bioequivalence limits, which can be viewed as target goalposts, are then used to determine BE by assessing whether or not LSM ratios and CIs fall within the targeted range.

Although such BE guidelines are generally appropriate for most drugs, the BE assessment of drugs with particular PK characteristics remains challenging. For example, the presence of endogenous levels for drugs such as iron or levothyroxine can make it difficult to distinguish between drug concentrations that come from exogenous sources and those already present in systemic circulation, especially if baseline levels account for a large portion of the observed concentrations $(18,19)$. Furthermore, endogenous substances are frequently subject to processes not typically associated with non-endogenous products (feedback mechanisms $(20,21,22)$, saturable transport or elimination, etc.) (23).

For iron products, the Office of Generic Drugs (OGD) of the US Food and Drug Administration (FDA) has recommended to sponsors of generic submissions that bioequivalence be assessed on baseline-adjusted $\mathrm{Cmax}_{\text {and }} \mathrm{AUC}_{0-\mathrm{t}}$ of total serum iron and of transferrin-bound iron. Considering the relative bioavailability of two iron formulations theoretically cannot be determined reliably by noncompartmental methods using total serum iron and transferrin-bound iron because of the changing baseline following iron administration and its nonlinear PK, other approaches for calculating AUC and Cmax and subsequently establishing the 
bioequivalence of iron products should be considered. Thus, we postulated that compartmental analyses, rather than noncompartmental analyses, could be more powerful (e.g., would present better statistical power for the same number of subjects) to use to determine the PK parameters necessary for the assessment of the relative bioavailability of two formulations of intravenously-administered Sodium Ferric Gluconate Complex in Sucrose (SFGC) simply because the non-linear characteristics of iron and its changing baseline could be addressed with that method. In other words, the aim of this analysis was to assess the relative bioavailability of two IV formulations of iron by using the compartmental approach. Standard noncompartmental analyses were also conducted so that statistical power would be compared, including results from a previously published study.

\section{METHODS}

\section{Study Design}

This study was an open label, randomized, single dose, two-treatment, two-period, two-sequence, crossover. A four week washout period was observed between doses. Subjects received both the test product (Sodium Ferric Gluconate Complex in Sucrose Injection, $62.5 \mathrm{mg} / 5 \mathrm{~mL}$ ) and the reference product (Ferrlecit ${ }^{\circledR}$ Injection by Watson Laboratories Inc., $62.5 \mathrm{mg} / 5 \mathrm{~mL}$ ) at a dose of 62.5 $\mathrm{mg}$ as an intravenous infusion over 30 minutes. Both treatments were diluted in $50 \mathrm{~mL}$ of $0.9 \%$ $\mathrm{NaCl}$.

Subjects were advised to fast for at least 10 hours before dosing, and until at least 4 hours after dosing. Water was permitted ad libitum. Standardized meals (with no specific restrictions on iron content) were served at about 4 (breakfast), 10 (lunch) and 14 (dinner) hours post-dose on Day 1 and at 24 (breakfast), 28 (lunch) and 33.5 (dinner) hours post-dose on Day 2.

Subjects were confined to the clinical facility from at least 10 hours before Day 1 dosing in each period and were required to stay until 36 hours thereafter. Subjects returned to the clinical site for the $48( \pm 1)$ and $72( \pm 1)$ hour post-dose blood sample collection.

During both periods, twenty one (21) PK samples were collected at the following times based on the start of the infusion: $-0.5,-0.25$ and -0.083 hour (to establish baseline values); 0.25 hour (midpoint of infusion), 0.5 hour (immediately at the end of the infusion); and $0.75,1,1.5,2,2.5,3,3.5,4,6$, $8,12,16,24,36,48$ and 72 hours after the start of the infusion. An additional $12.5 \mathrm{~mL}$ of blood was obtained from subjects pre-infusion and an additional $8.5 \mathrm{~mL}$ of blood was obtained from subjects at $4,16,48$ and 72 hours post-infusion start for the measure of hematocrit (only pre-infusion), ferritin, transferrin saturation and total iron binding capacity. A total of about $420 \mathrm{~mL}$ of blood was obtained from each subject over the course of the study for analysis.

Adverse events and vital signs were monitored throughout the study. Subjects were closely supervised and remained within sight of study personnel for four hours after receiving their initial dose. In addition, seated blood pressure and heart rate were measured prior to dosing and at 12, 24 and 36 hours post-dose.

\section{Population}

The study population was comprised of healthy male and non-pregnant female volunteers between the ages of 18 to 55 years old, inclusively, with low but normal iron levels. Ferritin levels had to be between 22 and $100 \mathrm{ng} / \mathrm{mL}$, inclusively, for men, and between 10 and $100 \mathrm{ng} / \mathrm{mL}$, inclusively, for women prior to first dosing. All subjects were required to have a body mass index of $18-32 \mathrm{~kg} / \mathrm{m}^{2}$ as well as an acceptable medical history, laboratory evaluation and physical examination before study entry. The laboratory tests included screens for biochemistry, hematology, urinalysis, cotinine, drugs of abuse, hepatitis B and C, and HIV as well as $\beta$-CG (if applicable) and FSH (if applicable).

\section{Ethics}

The protocol, protocol amendments and informed consent forms were approved by an institutional review board before any study-related procedures were initiated. Written, informed consent was obtained from volunteers prior to their participation in this study. This study was conducted in accordance with ethical principles outlined in the Declaration of Helsinki, and the TCPS: Ethical Conduct for Research Involving Humans as well as Canadian Regulatory requirements and guidelines.

\section{Sample Handling and Bioanalytical Method}

Samples were sequentially collected by direct venipuncture or catheter and processed in a timely manner. Samples were allowed to clot at room temperature for a minimum of 30 minutes and for a 
maximum of 45 minutes. The tubes were then centrifuged at $3000 \mathrm{RPM}$ and $4 \pm 1^{\circ} \mathrm{C}$ for 10 minutes and then placed at room temperature for a maximum of 15 minutes. A minimum of $1 \mathrm{~mL}$ of serum was transferred into duplicate $5 \mathrm{~mL}$ polypropylene tubes and maintained in the ice bath or cooling device until frozen. Samples were stored at approximately $-20^{\circ} \mathrm{C}$ (between -10 and $-35^{\circ} \mathrm{C}$ ) until transfer or shipment to the bioanalytical laboratory. The time between sample collection and freezer storage did not exceed 1.5 hours.

A validated analytical colorimetric method was used to assay total iron (TI) and transferrin bound iron (TBI). TI and TBI concentrations were measured within the validated standard curve range of 50.00 to $2000 \mathrm{mcg} / \mathrm{dL}$. Assays were performed by Cetero Research.

\section{Population Pharmacokinetic Modeling}

Datasets included subjects who completed the study and who had measurable concentrations of TI and TBI. Actual doses, infusion durations and sampling times were used to create the datasets, and iron lost through blood sampling was also taken into consideration. For each subject, the hematocrit value used to account for iron lost during blood sampling was calculated as an average of the hematocrit values taken before dosing in either Period 1 or 2 . Concentration values that were below the limit of quantitation (BLQ) were treated as missing values.

Compartmental analyses were performed using the software ADAPT $5{ }^{\circledR}$ (24), first using the maximum likelihood method to obtain initial estimates and subsequently using the iterative two stage (ITS) approach. This is a fully automated mixed effect modeling approach using both maximum likelihood and maximum a posteriori (MAP) modeling approaches. Briefly, the first probable population PK parameters and variance estimates (e.g., residual variability) were found by using maximum likelihood. Then a population analysis is undertaken where population, individual and residual variability PK parameters are calculated and updated with ever more probable values at every new population iteration. The mixed modeling approach was eventually stopped when it had converged (last iteration preceded by 10 consecutive iterations having a similar objective function (within 3\%)) and the most probable and stable results were found.
The base model used for model discrimination was a previously published multicompartmental model that simultaneously described the time courses of total iron, transferrin-bound iron and iron bound to sodium ferric gluconate complex (SFCGI) (25). The primary PK parameters estimated by this model included: $\mathrm{CL}_{1}$ (the clearance of SFGC-I to the RES), $\mathrm{CL}_{2}$ (clearance of SFGC-I directly to transferrin), $\mathrm{CL}_{3}$ (clearance of iron entering and exiting the marrow and red blood cell compartment), $\mathrm{CL}_{4}$ (clearance of TBI to the RES), $\mathrm{Km}$ (Iron concentration associated with half of the maximal rate of exchange between the RES and TBI compartments), Vss (the apparent steady-state volume of distribution of SFGC-I), V_RES (volume of distribution associated with the RES), V_RBC (marrow and red blood cell compartment), $\bar{V}_{-}$TBI (volume of distribution associated with TBI), and Vmax (maximal rate of exchange between the RES and TBI compartments).

All iron concentrations were fitted using weighting procedures of $\mathrm{W}_{\mathrm{j}}=1 / \sigma_{\mathrm{j}}^{2}$ where the variance $\sigma_{j}^{2}$ was calculated for each observation using the equation $S_{j}{ }^{2}=\left(a+b \cdot Y_{j}\right)^{2}$ where $a$ and $b$ are the intercept and slope of each variance model. The slope is the residual variability proportional to each concentration and the intercept is the additional component of the residual variability. Inter-subject variability was also estimated for each PK parameter estimated by the model.

Secondary PK parameters that were derived from the primary $\mathrm{PK}$ parameters included the following: CL (total clearance for SFGC-I, calculated as the sum of $\mathrm{CL}_{1}+\mathrm{CL}_{2}$ ), $\mathrm{AUC}_{\text {pred }}$ (area under the serum-time curve of SFGC-I, from the beginning of the infusion to infinity, calculated as dose divided by CL), $\mathrm{Cmax}_{\text {pred }}$ (maximum predicted serum concentration of serum SFGC-I over the 72hour sampling period), and $\mathrm{T}_{1 / 2}$ (apparent first-order terminal elimination half-life of SFGC-I).

In addition to standard metrics used to evaluate goodness of fit, visual predictive checks were performed. With the final model estimates for both population PK parameters and variability, concentration-time profiles for 1000 subjects were simulated, and median concentrations along with 95\% confidence intervals were established using the predicted concentrations. Observed concentration values and predicted confidence intervals were then overlaid graphically. 


\section{Statistical Analyses for Bioequivalence Assessment}

Analyses of variance (ANOVA) were performed on the natural logarithm of $\mathrm{Cmax}_{\text {pred }}$ and $\mathrm{AUC}_{\text {pred }}$ for SFGC-I obtained from the compartmental analysis. The ANOVA model included group, sequence, period nested within group and formulation as fixed effects and subject nested within group*sequence as a random effect. The group*formulation interaction was tested at a 5\% level of significance and removed from the model if it was not significant. Sequence was tested using subject nested within group*sequence as the error term. A 10\% level of significance was used to test the sequence effect. Each analysis of variance included calculation of least-squares means, the difference between adjusted formulation means and the standard error associated with this difference. The above statistical analyses were conducted using the appropriate $\mathrm{SAS}{ }^{\circledR}$ procedure.

In agreement with the two one-sided test for bioequivalence (26), 90\% confidence intervals for the difference between drug formulation leastsquares means (LSM) were calculated for $\mathrm{AUC}_{\text {pred }}$ and $\mathrm{Cmax}_{\text {pred }}$ obtained from the compartmental analysis, using the data transformed to their natural logarithm. The confidence intervals were expressed as a percentage relative to the LSM of the reference formulation.

Ratios of means were calculated using the LSM for the above mentioned $1 n$-transformed $\mathrm{AUC}_{\text {pred }}$ and $\mathrm{Cmax}_{\text {pred }}$ obtained from the compartmental analysis. The geometric mean values were reported and ratios of means were to be expressed as a percentage of the LSM for the reference formulation.

Bioequivalence was to be declared if the Test/Reference ratios of geometric means of $\mathrm{Cmax}_{\text {pred }}$ and $\mathrm{AUC}_{\text {pred }}$ and their complete $90 \%$ confidence intervals were to be contained within the bioequivalence interval 80.00 to $125.00 \%$ for iron bound to the Sodium Ferric Gluconate Complex.

\section{Noncompartmental Analyses}

Baseline-adjusted PK parameters $\mathrm{AUC}_{0-\mathrm{t}}$, AUCinf, Cmax and Tmax were calculated for TI and TBI. Baseline adjustments were performed by subtracting each individual's baseline value (which was the average of all 3 pre-dose values) from each of their post-dose concentration value. ANOVA were conducted on $\ln$-transformed PK parameters $\mathrm{AUC}_{0-\mathrm{t}}, \mathrm{AUCinf}$ and Cmax for TI and TBI using the same statistical model as the one employed for the parameters obtained from the compartmental analysis. Similarly, ratios of LSM and $90 \%$ CI were calculated for each parameter.

Results derived from noncompartmental analyses were also obtained from a previously published study (27). This study was an open-label, randomized, single-dose, parallel-group study conducted in 240 healthy volunteers under fasting conditions. Subjects received $125 \mathrm{mg}$ of the test (Nulecit $^{\mathrm{TM}}$, Watson Pharmaceuticals) or reference (Ferrlecit $^{\text {TM }}$, A. Nattermann \& Cie. GmbH.) SFGC formulation infused intravenously over 10 minutes. Samples for TI and TBI analysis were collected prior to dosing and at $0.0833,0.167,0.25,0.5,0.75$, $1,1.5,2,2.5,3,3.5,4,6,8,12,16,24$, and 36 hours after the start of the infusion. Samples were also collected at 24, 18, 12, 6, and 0 hours before dosing to determine baseline levels. Validated spectrophotometric assays were used to assay TI and TBI (refer to original publication for more details).

Post-dose concentration values were adjusted using the average of all 5 baseline values for each individual, and $\mathrm{PK}$ parameters $\mathrm{AUC}$ and Cmax were determined by standard noncompartmental methods with these baseline-adjusted concentrations assuming a stable baseline. ANOVA was conducted to compare $\ln$-transformed PK parameters between formulations. Treatment, group and group-by-treatment were used as classification variables. The group-by-treatment interaction and group terms were removed if they were deemed non-significant at an alpha level of 5\%. Geometric mean treatment ratios and the corresponding $90 \%$ CIs were determined and $\mathrm{BE}$ was declared if the ratios and $90 \%$ CIs were contained within 80 and $125 \%$ (27).

\section{RESULTS}

\section{Population Characteristics}

A total of 32 subjects were enrolled in the current study, and 29 completed both periods of the study. Subjects were dosed in two groups, where Group 1 (Subjects 1 to 16) was dosed on February 5, 2008 (Period 1) and March 4, 2008 (Period 2) and Group 2 (Subjects 17 to 32) was dosed on February 8, 2008 (Period 1) and March 7, 2008 (Period 2). Table 1 summarizes the demographic characteristics of the subjects enrolled in the study (both groups combined). 
Table 1. Demographic Traits of Subjects Included in the Population PK Analysis

\begin{tabular}{|c|c|}
\hline Characteristic & Number (\%) \\
\hline \multicolumn{2}{|l|}{ Sex } \\
\hline Female & $24(75 \%)$ \\
\hline Male & $8(25 \%)$ \\
\hline \multicolumn{2}{|l|}{ Race } \\
\hline Caucasian & $20(62.5 \%)$ \\
\hline African American & $7(21.9 \%)$ \\
\hline Asian & $4(12.5 \%)$ \\
\hline Native American & $1(3.1 \%)$ \\
\hline \multirow[t]{2}{*}{ Characteristic } & Mean \pm SD $(C V \%)$ \\
\hline & Median (Minimum - Maximum) \\
\hline \multirow[t]{2}{*}{ Age (years) } & $37.3 \pm 9.29(24.9 \%)$ \\
\hline & $37.5(22-51)$ \\
\hline \multirow[t]{2}{*}{ Height (cm) } & $164.4 \pm 7.20(4.38 \%)$ \\
\hline & $164.5(149.5-180.0)$ \\
\hline \multirow[t]{2}{*}{ Weight (kg) } & $67.8 \pm 10.3(15.1 \%)$ \\
\hline & $65.5(47.1-89.1)$ \\
\hline \multirow[t]{2}{*}{ Body mass index $\left(\mathrm{kg} / \mathrm{m}^{2}\right)$} & $25.0 \pm 3.00(12.0 \%)$ \\
\hline & $24.8(20.4-31.8)$ \\
\hline \multirow[t]{2}{*}{ Hemoglobin at screening $(\mathrm{g} / \mathrm{dL})$} & $13.4 \pm 1.09(8.13 \%)$ \\
\hline & $13.2(11.2-15.4)$ \\
\hline \multirow[t]{2}{*}{ Hematocrit at screening (\%) } & $40.3 \pm 3.35(8.32 \%)$ \\
\hline & $39.9(33.9-46.1)$ \\
\hline
\end{tabular}

Of the 3 subjects who did not complete the clinical phase of the study, 2 withdrew before Period 2 because of adverse events deemed unlikely to be related to the investigational product and one subject was withdrawn by the Investigator because of a positive drug screen test (amphetamines) at Period 2 check-in.

Subjects included in the previously published study were predominantly White (comprising around $91 \%$ of the subjects receiving the generic formulation and $85 \%$ of the subjects receiving the reference formulation). Around $7 \%$ and $13 \%$ of subjects in the generic and reference treatment arms, respectively, were Black or African American and other races (American Indian/Alaskan, Native Hawaiian/Pacific Islander, multirace subjects) accounted for less than $2.5 \%$ of subjects in both groups. In terms of ethnicity, over $90 \%$ of subjects in each arm were not Hispanic or Latino. In both treatment arms, around $50 \%$ of the subjects were male. Mean $( \pm$ SD) age, height, weight and body mass index in the generic cohort were $30.8 \pm 9.6$ years, $171 \pm 9.5 \mathrm{~cm}, 75.1 \pm 15.0 \mathrm{~kg}$ and $25.5 \pm 3.77$ $\mathrm{kg} / \mathrm{m}^{2}$, respectively. Mean $( \pm \mathrm{SD})$ values for age, height, weight and body mass index were similar in the reference cohort, at $29.9 \pm 8.5$ years, $172 \pm 8.7$ $\mathrm{cm}, 77.5 \pm 13.4 \mathrm{~kg}$ and $26.0 \pm 3.50 \mathrm{~kg} / \mathrm{m}^{2}$, respectively.

\section{Population PK Approach}

A total of 2413 concentrations of TI and TBI were included in the analysis. Two basic models were evaluated. In the first model, it was assumed that the test and reference product had the same values for $\mathrm{CL}_{1}, \mathrm{CL}_{2}$ and Vss but with a different relative bioavailability factor (Frel). In other words, $\mathrm{CL}_{1}$, $\mathrm{CL}_{2}$ and Vss between formulations only differed by the same factor Frel. In the second model, different values for $\mathrm{CL}_{1}, \mathrm{CL}_{2}$ and Vss were estimated for the test and reference products. Results from the STS analysis performed with these 2 models are presented in Table 2.

Based on the model discrimination criteria, especially as indicated by the Bayesian and Akaike information criterion, as well as the graphical indicators of goodness of fit, the first model was superior. Two additional parameters were estimated: C_NTBI_P1 and C_NTBI_P2. These parameters estimated the concentrations of nontransferrin bound iron in Periods 1 and 2, respectively. Differential equations describing the final model were: 


$$
\begin{gathered}
\frac{d X(1)}{d t}=R(1)-\frac{C L_{1}+C L_{2}}{V s S} \cdot X(1) \\
\frac{d X(2)}{d t}=\frac{C L_{1}}{V s S} \cdot X(1)+\frac{C L_{3}}{V_{-} R B C} \cdot X(4)+\frac{C L_{4}}{V_{-} T B I} \cdot X(3)-\frac{V_{\max }}{K m \cdot V_{-} R B C+X(2)} \cdot X(2) \\
\frac{d X(3)}{d t}=\frac{C L_{2}}{V S S} \cdot X(1)+\frac{V_{\max }}{K m \cdot V_{-} R B C+X(2)} \cdot X(2)-\frac{C L_{4}}{V_{-} T B I} \cdot X(3)-\frac{C L_{3}}{V_{-} T B I} \cdot X(3) \\
\frac{d X(4)}{d t}=\frac{C L_{3}}{V_{-} T B I} \cdot X(3)-\frac{C L_{3}}{V_{-} R B C} \cdot X(4)-K 0 \cdot R(2)
\end{gathered}
$$

Table 2. PK Model Discrimination

\begin{tabular}{cccccccc}
\hline Model & BIC & AIC & OF & \multicolumn{2}{c}{$\mathbf{R}^{\mathbf{2}}$} & \multicolumn{2}{c}{ Residual variability (\%) } \\
\hline & & & TI & TBI & TI & TBI \\
Model 1 & 990.742 & 945.014 & 453.507 & 0.927 & 0.808 & 21.0 & 17.2 \\
Model 2 & 998.591 & 948.050 & 453.025 & 0.930 & 0.829 & 20.1 & 16.0 \\
\hline \multicolumn{2}{l}{ AIC - Akaike information criterion; BIC - Bayesian information criterion; OF - Objective function; } \\
\hline
\end{tabular}

Where $X(1), X(2), X(3)$ and $X(4)$ represent the amount of iron in the serum, RES, TBI and red blood cell (marrow) compartments. R(1) represents the SFGC infusion rate while $\mathrm{R}(2)$ is an on/off switch that accounts for the iron loss associated with blood sampling.

The parameter Vmax was defined as $V \max =$ $\left(\mathrm{CL}_{3}+\mathrm{CL}_{4}\right) \mathrm{x}\left(\mathrm{Km}+\mathrm{TBI}_{\text {base }}\right)$, where $\mathrm{TBI}_{\text {base }}$ represents the observed baseline TBI concentration for each subject before dosing in each period. This equation was determined from the assumption that prior to the administration of SFGC, iron levels are at an equilibrium between the RES, TBI and RBC (marrow) compartments.

Observed concentrations for total serum iron and TBI, parameterized as $\mathrm{Y}(1)$ and $\mathrm{Y}(2)$, respectively, were fitted according to the following equations, where C_NTBI was different for Periods 1 and 2, as previously described.

$$
\begin{gathered}
Y(1)=\frac{X(1)}{V S S}+\frac{X(3)}{V_{-} T B I}+C_{-} N T B I \\
Y(2)=\frac{X(3)}{V_{-} T B I}
\end{gathered}
$$

The final model is depicted in Figure 1 and the PK parameter estimates from the final model are presented in Table 3. Plots of goodness of fit are presented in Figure 2 while visual predictive checks are presented in Figure 3.

As demonstrated by the goodness-of-fit plots and visual predictive checks, the model adequately describes all observed concentrations of total iron and transferrin-bound iron. Predicted versus observed concentrations were randomly scattered around lines of identity. No important trends were observed with respect to the standardized weighted residuals or with respect to time. In addition, the residual variability, which includes the intraindividual variability, variability from the bioanalytical measurement, all experimental error and all errors from the modeling itself, was only $23.0 \%$ and $17.2 \%$ for total iron and transferrinbound iron, respectively.

Secondary PK parameters estimated from the final model are presented in Table 4. The median estimated terminal elimination half-life for both the test and reference products was 1.63 hours (ranging from 0.785 to 5.92 hours).

ANOVA results demonstrated no statistically significant sequence effect at a $10 \%$ level, although there was a statistically significant group effect for ln-transformed $\mathrm{Cmax}_{\text {pred }}(\mathrm{p}=0.0104)$. Because the sizes of Groups 1 and 2 were similar $(n=15$ and $\mathrm{n}=14$, respectively), the equality of variances test was not performed since ANOVA is robust to the violation of the equality of variance assumption when groups are equally sized and larger than 5 (28). Statistical analyses on the ln-transformed $\mathrm{Cmax}_{\text {pred }}$ and $\mathrm{AUC}_{\text {pred }}$ parameters for SFGC-I are presented in Table 5.

\section{Noncompartmental Approach}

Mean concentration-time profiles for TI and TBI associated with each of the studies are presented in Figure 4. ANOVA results obtained from noncompartmental analyses of both studies are summarized in Table 5. 


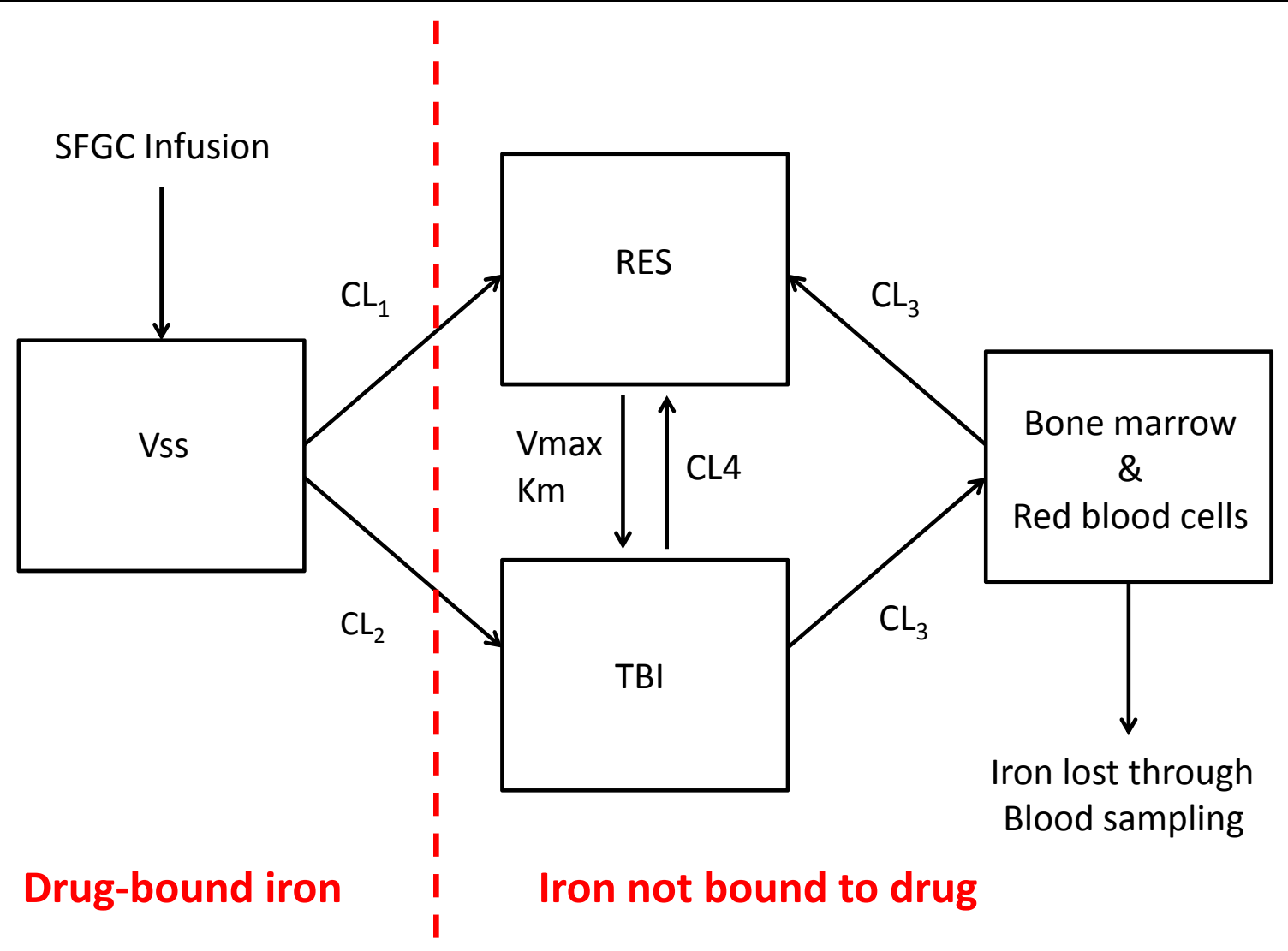

Figure 1. Final PK Model

Table 3. Population Pharmacokinetic Parameter Estimates

\begin{tabular}{|c|c|c|}
\hline PK Parameter & Mean (CV\%) & Median (Minimum- Maximum) \\
\hline $\mathrm{CL}_{1}(\mathrm{~L} / \mathrm{h})$ & $2.25(32.0 \%)$ & $2.03(1.46-4.17)$ \\
\hline $\mathrm{CL}_{2}(\mathrm{~L} / \mathrm{h})$ & $0.0458(11.8 \%)$ & $0.0455(0.0357-0.0571)$ \\
\hline Vss (L) & $4.41(14.5 \%)$ & $4.53(3.32-5.50)$ \\
\hline V_RES (L) & $1220(0.175 \%)$ & $1220(1214-1226)$ \\
\hline $\mathrm{V}_{-}^{-} \mathrm{TBI}(\mathrm{L})$ & $0.589(5.62 \%)$ & $0.595(0.522-0.637)$ \\
\hline $\mathrm{CL}_{3}(\mathrm{~L} / \mathrm{h})$ & $0.000000213(59.5 \%)$ & $0.000000267(4.01 \mathrm{E}-11-3.48 \mathrm{E}-07)$ \\
\hline V_RBC (L) & $0.000288(55.0 \%)$ & $0.000369(0.00000539-0.000451)$ \\
\hline $\mathrm{CL}_{4}(\mathrm{~L} / \mathrm{h})$ & $0.0313(23.2 \%)$ & $0.0315(0.0170-0.0439)$ \\
\hline $\mathrm{Km}(\mathrm{mcg} / \mathrm{dL})$ & $36.1(37.0 \%)$ & $38.8(3.61-59.7)$ \\
\hline Frel & $0.905(13.9 \%)$ & $0.926(0.644-1.19)$ \\
\hline C_NTBI_P1 (mcg/dL) & $44.3(52.8 \%)$ & $45.5(5.18-83.1)$ \\
\hline $\mathrm{C}^{-} \mathrm{NTBI} \mathrm{P} 2(\mathrm{mcg} / \mathrm{dL})$ & $75.8(50.2 \%)$ & $73.6(0.00-147)$ \\
\hline
\end{tabular}

$\mathrm{CL}_{1}$ : Clearance of SFGC-I to the reticuloendothelial system (RES) compartment; $\mathrm{CL}_{2}$ : Clearance of SFGC-I directly to transferrin; Vss: the apparent steady-state volume of distribution of SFGC-I; V_RES: volume of distribution associated with the RES; V_TBI: volume of distribution associated with TBI; $\mathrm{CL}_{3}$ : clearance of iron entering and exiting the marrow and red blood cell compartment; V_RBC: marrow and red blood cell compartment; $\mathrm{CL}_{4}$ : clearance of TBI to the RES; Km: Iron concentration associated with half of the maximal rate of exchange between the RES and TBI compartments; Frel: Relative bioavailability factor; C_NTBI_P1: Concentration of non-transferrin-bound iron during Period 1; C_NTBI_P2: Concentration of non-transferrin-bound iron during Period 2; 


\section{TOTAL IRON}

A

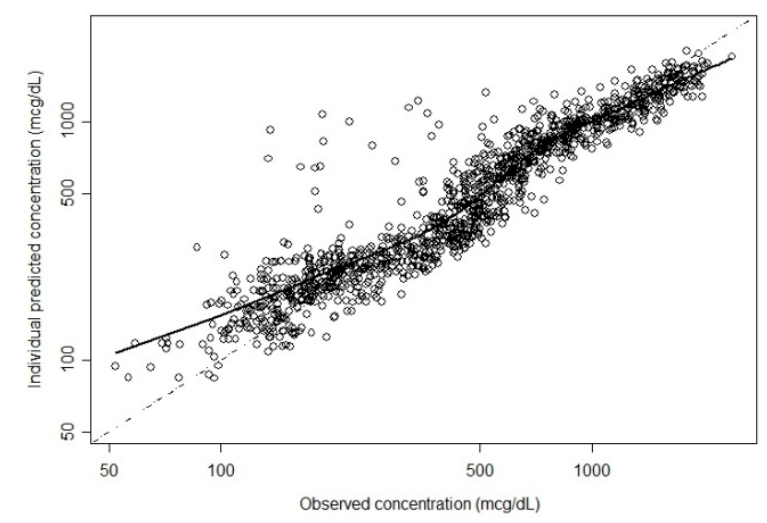

B

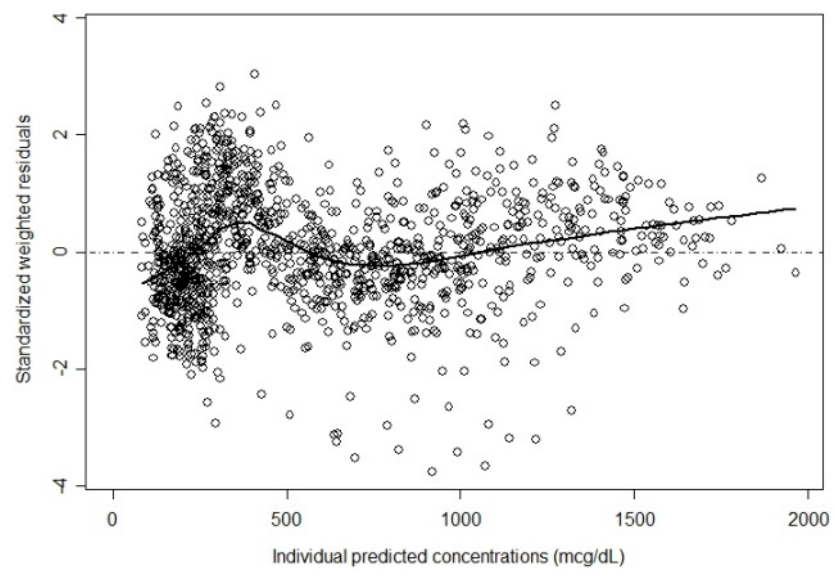

C

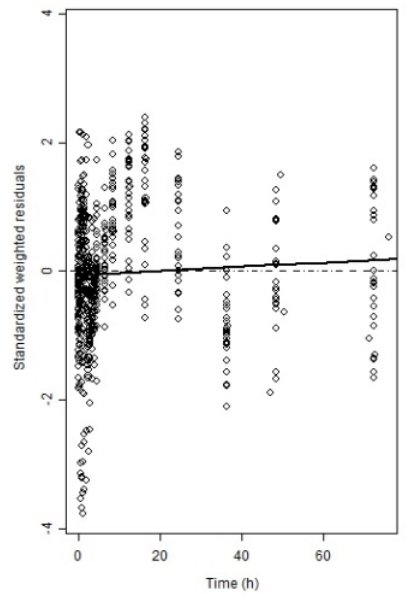

\section{TRANSFERRIN BOUND IRON}

\section{D}

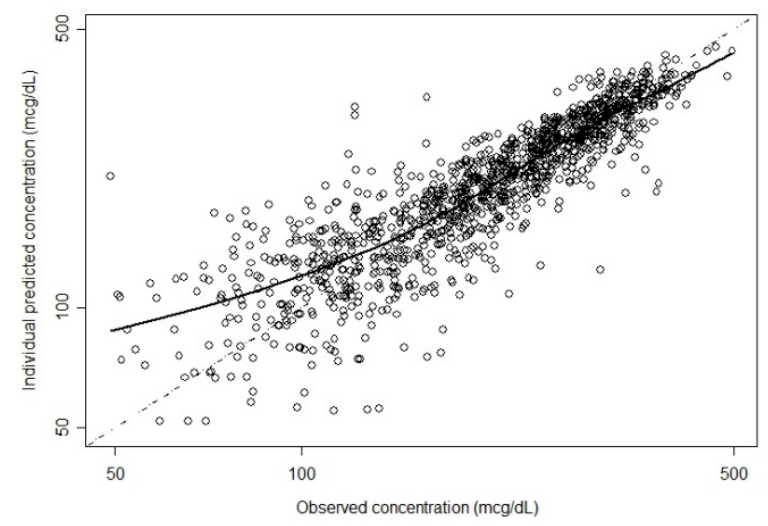

$\mathbf{E}$

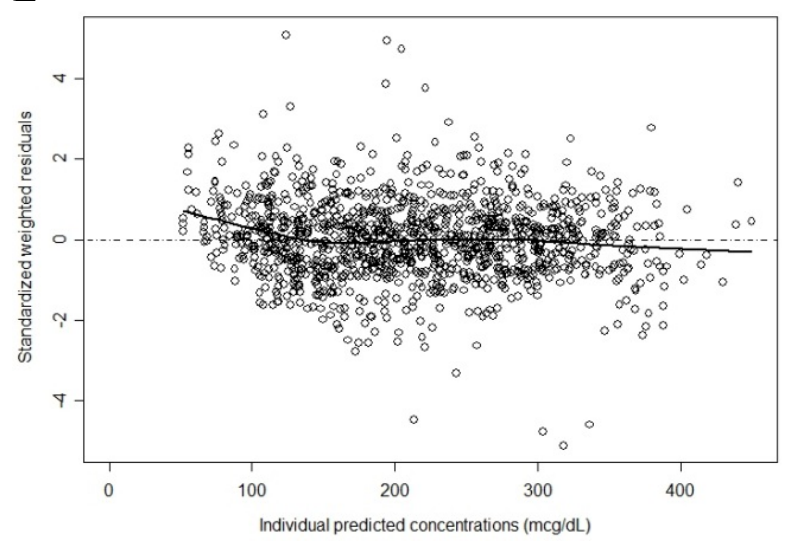

$\mathbf{F}$
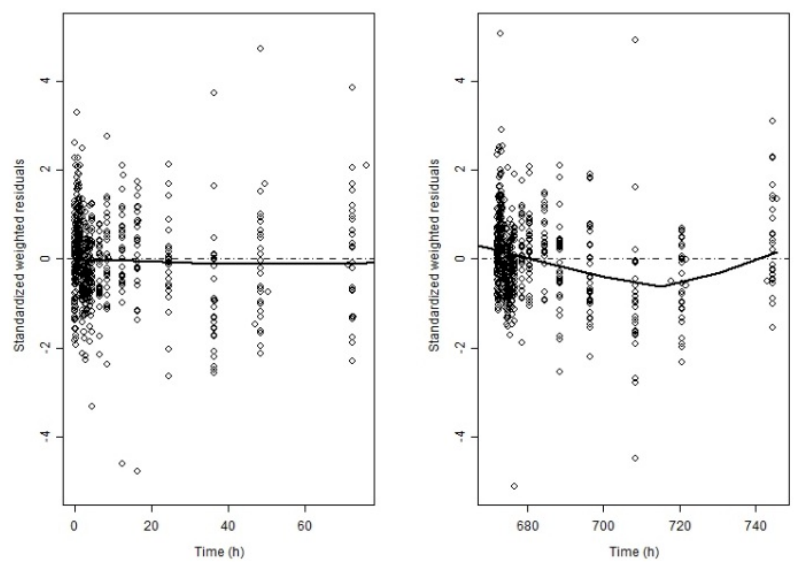

Figure 2. Goodness-of-Fit Plots Individual observed versus individual predicted total iron concentrations on a log scale (A); Standardized residuals versus individual predicted total iron concentrations (B); Standardized residuals versus time for total iron concentrations (C); Individual observed versus individual predicted transferrin-bound iron concentrations on a log scale (D); Standardized residuals versus individual predicted transferrin-bound iron concentrations (E); Standardized residuals versus time for transferrin-bound iron concentrations (F); Legend: Circles $=$ observed concentrations, Dotted line $=$ reference line (unity or zero), Solid black line $=$ Loess curve $(\operatorname{span}=0.5$, degree $=1)$ 
TOTAL IRON

A

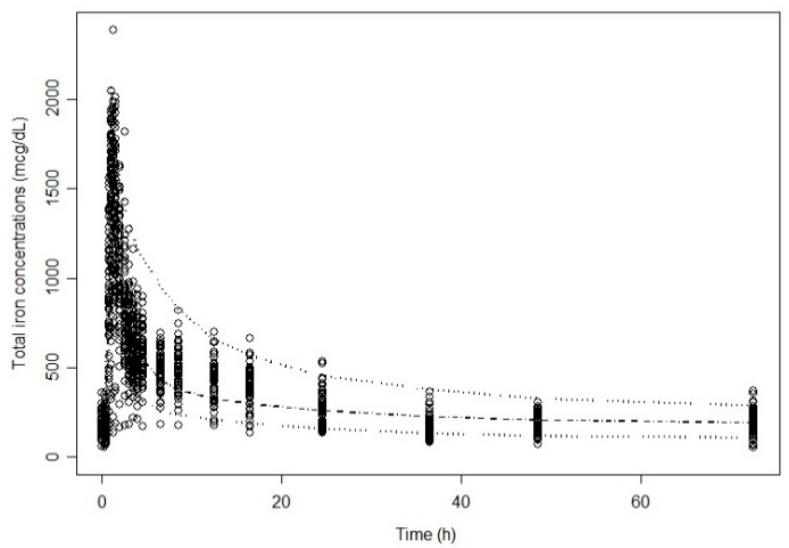

B

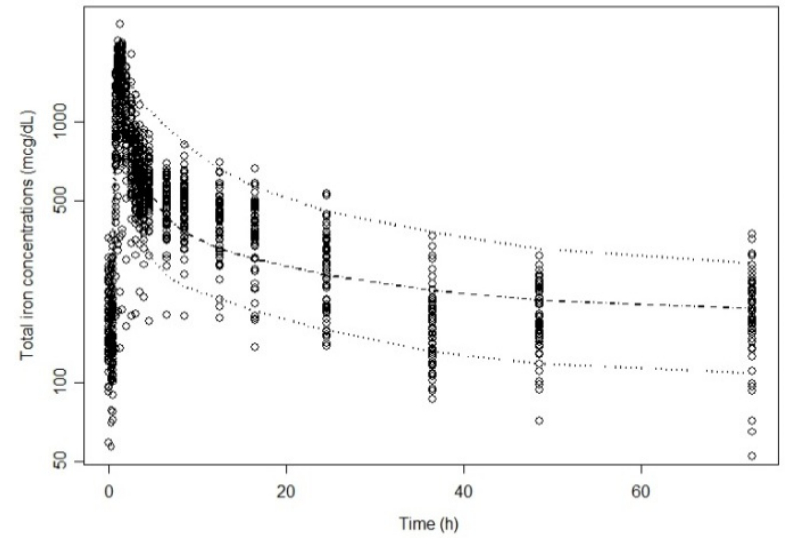

TRANSFERRIN BOUND IRON

C

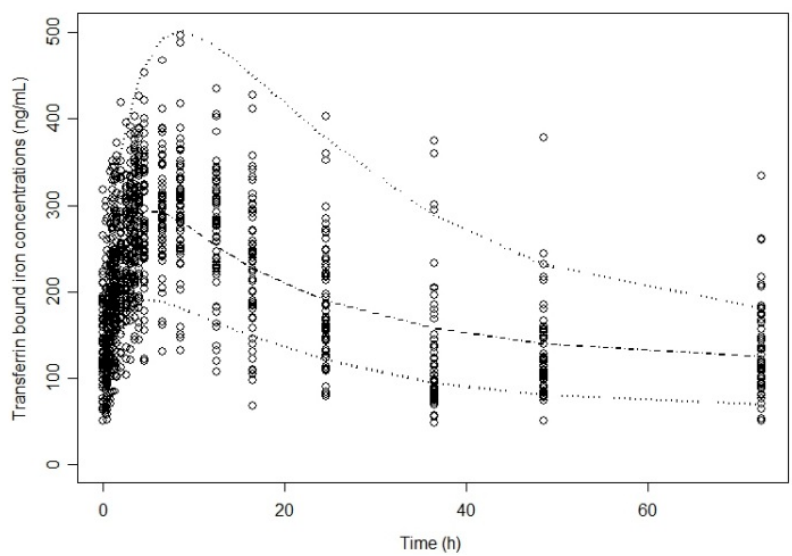

D

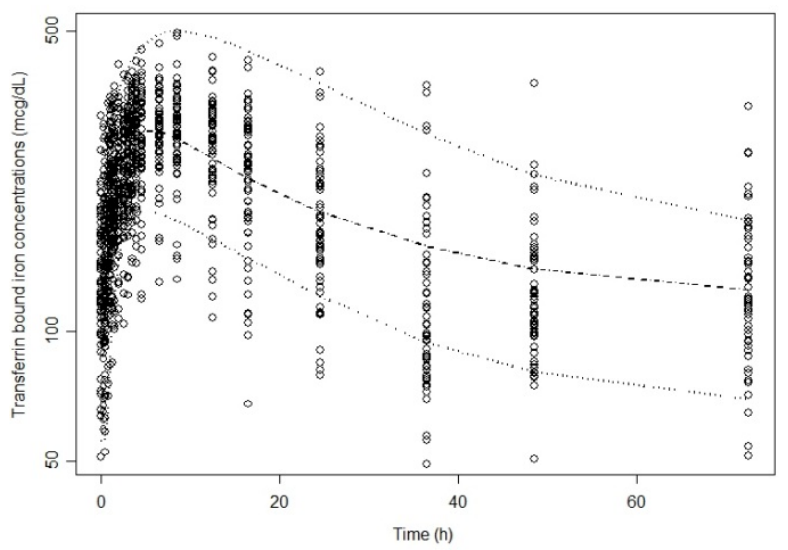

Figure 3. Visual Predictive Checks Total Iron Concentration Time Profiles (Linear Scale) (A); Total Iron Concentration Time Profiles (Semi-Log Scale) (B); Transferrin Bound Iron Concentration Time Profiles (Linear Scale) (C); Transferrin Bound Iron Concentration Time Profiles (Semi-Log Scale) (D); Legend: Circles = observed concentrations; Dotted line = $95 \%$ confidence intervals; Dashed line $=$ median predicted concentration .

Table 4. Secondary PK Parameters

\section{Parameter}

Mean (CV\%) Median (Range)

Test

Vss (L)

$\mathrm{CL}(\mathrm{L} / \mathrm{h})$

$\mathrm{AUC}_{\text {pred }}(\mathrm{mcg} * \mathrm{~h} / \mathrm{dL})$

$\mathrm{Cmax}_{\text {pred }}(\mathrm{mcg} / \mathrm{dL})$

$4.96(19.3 \%)$
$5.20(3.38-6.41)$
$2.60(34.4 \%)$
$2.30(1.55-4.77)$
$2662(30.8 \%)$
$2714(1400-4033)$
$1150(20.5 \%)$
$1100(854-1654)$

\section{Reference}

$4.41(14.5 \%)$

$4.53(3.32-5.50)$

$2.30(31.4 \%)$

$2.07(1.51-4.22)$

$2927(24.9 \%)$

$3017(1481-4152)$

$1270(14.7 \%)$

$1227(946-1649)$ 


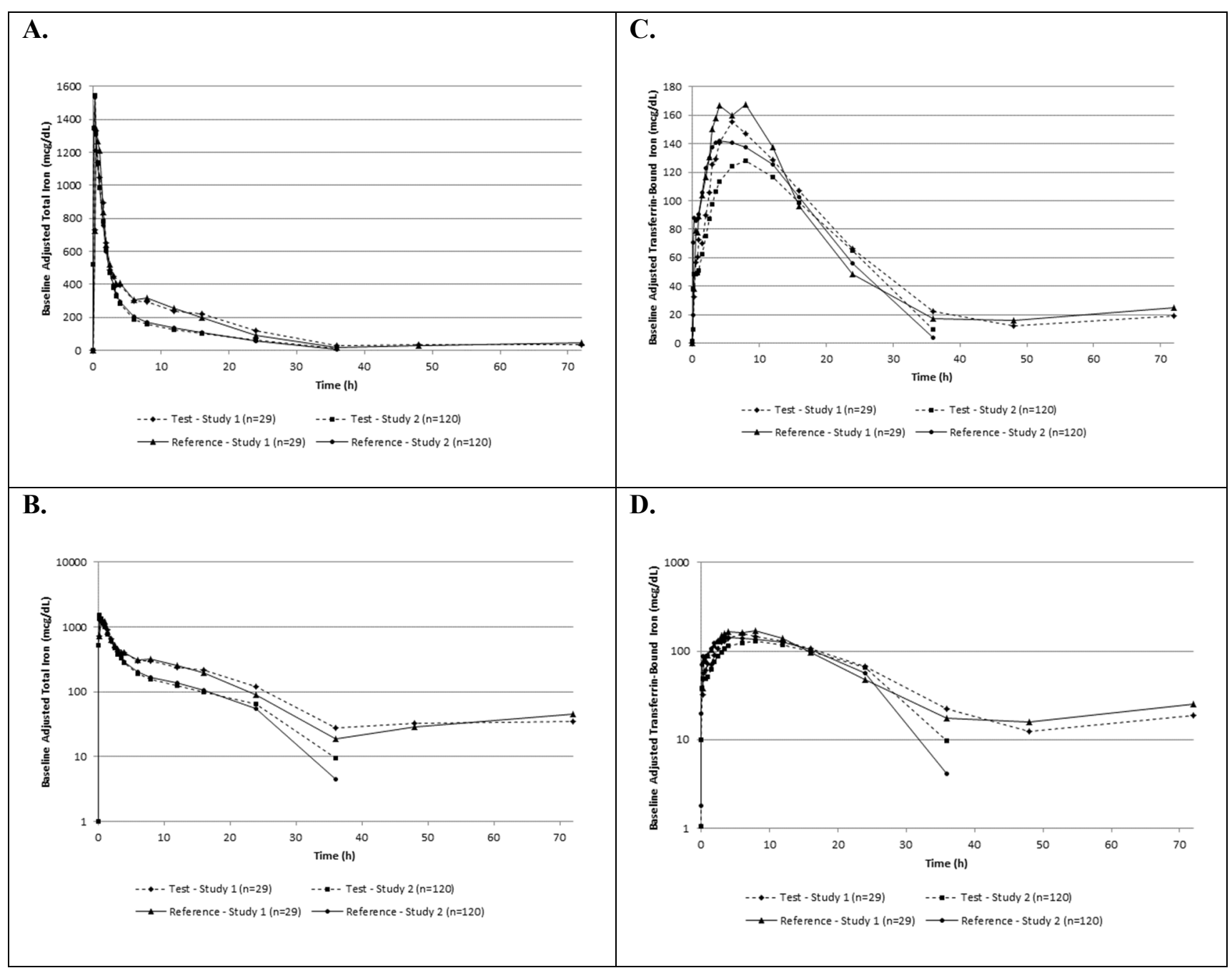

Figure 4. Mean Concentration Versus Time Profiles (A) Baseline-adjusted total iron profiles (linear scale) (B) Baselineadjusted total iron profiles (semi-log scale) (C) Baseline-adjusted transferrin-bound iron profiles (linear scale) (D) Baselineadjusted transferrin-bound iron profiles (semi-log scale); Legend: Dashed lines represent the test product while solid lines represent the reference product. Diamond and triangle symbols represent data associated with Study 1 while squares and circles are associated with Study 2.

\section{DISCUSSION}

Both the standard, noncompartmental approach to $\mathrm{BE}$ assessment (only when $\mathrm{n}=240$ ) as well as the innovative population $\mathrm{PK}$ approach $(\mathrm{n}=29)$ demonstrated $\mathrm{BE}$ between the iron formulations that were tested. To our knowledge, this is the first published account of compartmental analyses being used to demonstrate $\mathrm{BE}$ with pharmacokinetic endpoints for iron products. This unconventional approach was favoured over the traditional noncompartmental method of calculating Cmax and AUC because of the particularities of iron pharmacokinetics. Noncompartmental analyses are robust when certain assumptions hold true, and iron violates many of these assumptions.

The first assumption is that the drug in question displays linear pharmacokinetics $(10,12)$. In other words, exposure increases in proportion with increasing dose. A second important assumption is that the drug is eliminated strictly from the body from the pool in which it is being measured, the plasma, for example and in a continuous fashion $(11,12)$. Finally, this approach assumes that all sources of the drug are direct and unique to the measured pool, and that consequently baseline levels remain constant (12). In contrast, the compartmental method employed in the current 
analysis does not require the drug under study to meet such assumptions as the model included all of these iron PK characteristics. Similar approaches could be applied to analyze other difficult drugs from a PK point of view. Examples may include enzymes or metabolites that are metabolized intracellularly (29).

Another challenge for assessing SFGC pharmacokinetics is the inability to assay the iron associated with SFGC directly. Although it is possible to assay total serum iron and transferrinbound iron, it is impossible to distinguish endogenous iron from the iron provided by SFGC supplementation. One approach that is often used to work around this problem involves subtracting transferrin-bound iron levels from total serum iron levels, thus assuming that the resulting concentration differences represent iron from the SFGC. In contrast, the compartmental model used in the current analysis estimates iron levels coming from both endogenous and exogenous sources without performing this simple subtraction and does not assume that total iron is only composed of TBI and SFGC-I.

In order to apply compartmental analysis in a $\mathrm{BE}$ context, the first step was the determination of a compartmental model that described the pharmacokinetics of iron in all its forms (total, bound to transferrin and associated with SFGC). Based on a previously published model (25), a model describing both formulations of iron was established. Overall, it explained the data very well. Although twelve parameters were estimated by the model, the model was not deemed overparameterized since two analytes were being fitted, each demonstrating two visible exponentials in their disposition with known nonlinearity (30). In addition, over 80 concentrations were fitted per subject, signifying that 6 samples were available per PK parameter, which represents a clear rich sampling scenario for the PK model (sparse sampling can be defined when less than 1 sample is available per fitted PK parameter). This PK model accounted for serum iron, iron bound to transferrin, and stores in the reticuloendothelial system and bone marrow (red blood cells). The model also took into consideration iron lost during each blood sample, as well as iron not bound to transferrin. Although the administration of intravenous iron is not associated with the generation of detectable or dialyzable free iron $(3,31)$, there is evidence that points to the existence of non-transferrin bound iron
(NTBI) that is biologically active and labile $(9,31,32)$. This NTBI may even be bound to albumin (32). The levels of NTBI estimated in our population were about $46 \mathrm{mcg} / \mathrm{dL}$ before any treatment (Period 1) and about $74 \mathrm{mcg} / \mathrm{dL}$ before Period 2, which are equivalent to roughly 0.001 and $0.002 \mathrm{mcM}$ and which are well under the $1 \mathrm{M}$ levels normally seen in healthy subjects (1).

The PK parameters for volume of distribution and clearance estimated with our model were slightly lower than what has been reported in the literature. The Vss and CL estimated for SFGC-I from our analysis were $4.41 \mathrm{~L}$ and $2.3-2.6 \mathrm{~L} / \mathrm{h}$, whereas they were $5.72 \mathrm{~L}$ and $3.87 \mathrm{~L} / \mathrm{h}$ in the previous study for which a compartmental analysis was used (25). Conversely, the average SFGC-I half-life in our study was $1.63 \mathrm{~h}$ whereas it was closer to $1 \mathrm{~h}$ in the other study (25). The differences observed between the PK parameter estimates from our analysis and those in the literature may result from the differences in study populations. In the current study, enrolled subjects had ferritin levels between $22 \mathrm{ng} / \mathrm{mL}$ (10 ng/mL for women) and 100 $\mathrm{ng} / \mathrm{mL}$, inclusively, whereas levels were less than or equal to $20 \mathrm{ng} / \mathrm{mL}$ in the other study. This means that the subjects in this analysis had a less profound iron deficiency than those studied by Seligman et al., considering ferritin is a measure of bodily iron stores (9). Degree of deficiency has been shown to influence iron pharmacokinetics, particularly its rate of transfer from RBC to RES (4). Patients who are more iron-deficient incorporate iron faster into the RES, which could explain why the terminal elimination half-life of SFGC-I determined by others was shorter than in the current study. In addition, the proportion of women was different in each study. The study population in the current study was composed of $75 \%$ women whereas women only made up $43 \%$ of the other population. As iron storage and loss are different between men and women (9), it is possible that the different gender compositions led to slightly different pharmacokinetic parameter estimates.

By using a compartmental approach to assess the BE of two formulations of SFGC, problems associated with the noncompartmental method were altogether avoided. Indeed, because SFGC-I Cmax and AUC were calculated by model-based methods, iron's non-linear behaviour, non- continuous elimination (e.g., only through the specific blood samples), unstable baseline, and continual recycling were no longer issues as they were directly 
addressed by the model. All the specificity of iron pharmacokinetics such as non-transferrin bound iron and iron lost through blood draws were specifically incorporated. The absence of a stable baseline for both total iron and TBI also became a non-issue as the model fitted all the analytes specifically and allowed for the fact that the iron administration in the two periods naturally raised the levels of TBI and total serum iron. The overall result is that all of this unaccountable variability in the baseline adjusted concentrations of total serum iron and TBI are not present in the population compartmental analysis, therefore demonstrating $\mathrm{BE}$ with a much lower number of subjects because of this lower unaccountable variability.

The population PK modeling approach described in this article shows that BE can be demonstrated with a relatively small sample size for iron products. Although BE was concluded in the study described by Baribeault (27), it was necessary to dose 240 subjects in a parallel-group design. Moreover, when noncompartmental analyses were conducted with data from our study, only one parameter (Cmax of baseline-adjusted TBI) met bioequivalence criteria. Post-hoc analyses also revealed that the study lacked the power to show equivalence at $\pm 20 \%$ with an alpha error of $5 \%$ (i.e., power was less than $40 \%$ ). Overall, this suggests that in order to demonstrate $\mathrm{BE}$ between SFGC formulations using traditional noncompartmental methods, a very large number of subjects must be dosed. In contrast, the compartmental approach was adequately powered to show equivalence with a sample size of 29 subjects. This highlights again the strengths of the compartmental approach used in this study, as it is able to demonstrate bioequivalence with a significantly smaller number of subjects in a crossover design. In other words, this approach is not only scientifically sound, but it decreases the number of subjects who are exposed to the study drugs, resulting in a more cost-efficient and timeefficient study.

Other researchers have shown, through the analysis of simulated data or data obtained from real clinical trials, that the compartmental approach can be used to assess bioequivalence $(33,34,35,36,37,38,39,40,41)$. However, the PK models employed in previously published analyses were relatively simple ones (one or two compartment models). The population PK model developed in the current analysis is obviously more complex, but it allowed us to simultaneously characterize the PK of two different analytes (TI, TBI) and present concentration time profiles for what was directly administered ferrlecit-bound-iron or SFGC.

The analysis presented here demonstrates how the compartmental approach can be used to perform $\mathrm{BE}$ assessments for drugs that do not meet assumptions necessary to employ more traditional, noncompartmental approaches. These drugs may or may not be highly variable drugs (i.e., drugs for which within-subject variability of AUC or Cmax are greater than $30 \%$ ). Thus, for highly variable drugs that do not lend themselves to traditional analyses, the compartmental approach described here can be adopted. This would first entail developing a model that simultaneously describes the PK of both the test and reference drugs, which could be based on models already described in the literature. Specific model development criteria must be established a priori in the protocol and ideally in a population PK analysis plan. For highly variable drugs that meet the criteria associated with standard noncompartmental analyses, use of the compartmental approach can also be used but other types of analyses, such as the reference-scaled average bioequivalence method $(42,43)$, should also be considered.

Although there were many advantages to adopting this non-traditional approach to evaluating $\mathrm{BE}$, the development of a PK model for iron was more time-consuming and labour-intensive than the noncompartmental approach from an analysis perspective. The advantages and disadvantages of using a compartmental PK approach for a highly variable drug that meets the assumptions associated with standard analyses must be weighed in comparison with the reference-scaled average bioequivalence approach. Despite this, results show that this compartmental approach to BE assessment should be seriously considered for iron and potentially other rare, complicated drugs from a PK point of view for which traditional methods are unsuitable.

\section{CONCLUSIONS}

A compartmental analysis approach was applied successfully to demonstrate the BE between two formulations of sodium ferric gluconate complex in sucrose. BE was also concluded for the same products in a separate, much larger, parallel-design 
study employing traditional, noncompartmental methods of analysis. The results of this study suggest that alternative methods, such as the population compartmental analysis proposed here, should also be considered for assessing BE of drugs that are complicated from a PK point of view and for which the standard approach becomes artificially variable thereby necessitating the enrollment of too many subjects.

\section{ACKNOWLEDGMENTS}

The studies described in this manuscript were sponsored by Watson Laboratories Inc.

\section{REFERENCES}

1. Chua A, Graham R, Trinder D, Olynyk J. The regulation of cellular iron metabolism. Critical reviews in clinical laboratory sciences. 2007; 44(56): p. 413-59.

2. Wood R, Ronnenberg A. Iron. In Shils M, Shike M, Ross A, Caballero B, Cousins R, editors. Modern Nutrition in Health and Disease. 10th ed. Philadelphia: Lippincott Williams \& Wilkins; 2006.

3. Van Wyck D, Danielson B, Aronoff G. Making sense: a scientific approach to intravenous iron therapy. Journal of the American Society of Nephrology. 2004; 15(Suppl 2): p. S91-2.

4. Danielson B. Structure, chemistry, and pharmacokinetics of intravenous iron agents. Journal of the American Society of Nephrology. 2004; 15(Suppl 2): p. S93-8.

5. Knutson $M$ WRM. Iron metabolism in the reticuloendothelial system. Critical reviews in biochemistry and molecular biology. 2003; 38(1): p. 61-88.

6. Adamson J. Iron Deficiency and Other Hypoproliferative Anemias. In Fauci A, Braunwald E, Kasper D, Hauser S, Longo D, Jameson J, editors. Harrison's Principles of Internal Medicine. 18th ed.: The McGraw-Hill Companies, Inc.; 2012.

7. Andrews N. Iron Deficiency and Related Disorders. In Greer J, Foerster J, Rodgers G, Paraskevas F, Glader B, Arber D, editors. Wintrobe's Clinical Hematology. 12th ed. Philadelphia: Lippincott Williams \& Wilkins; 2009.

8. Henderson P, Hillman R. Characteristics of iron dextran utilization in man. Blood. 1969; 34(3): p. $357-75$.

9. Clark S. Iron deficiency anemia. Nutrition in clinical practice. 2008 ; 23(2): p. 128-41.

10. Gibaldi M, Perrier D. Noncompartmental Analysis
Based on Statistical Moment Theory. In Gibaldi M, Perrier D, editors. Pharmacokinetics. 2nd ed. New York: Informa Healthcare USA, Inc.; 2007. p. 40916.

11. Benet L, Ronfeld R. Volume terms in pharmacokinetics. Journal of pharmaceutical sciences. 1969; 58(5): p. 639-41.

12. DiStefano JI, Landaw E. Multiexponential, multicompartmental, and noncompartmental modeling. I. Methodological limitations and physiological interpretations. Am J Physiol. 1984;: p. R651-64.

13. U.S. Food and Drug Administration. Bioavailability and Bioequivalence Requirements. 2011. CFR 21.320.1.

14. U.S. Food and Drug Administration. Guidance for Industry: Bioavailability and Bioequivalence Studies for Orally Administered Drug Products - General Considerations. Guidance document. Rockville:, Health and Human Services; 2003.

15. Committee for Medicinal Products for Human Use, European Medicines Agency. Guideline on the Investigation of Bioequivalence. Guidance document. London:; 2010.

16. Therapeutic Products Directorate, Health Canada. Draft Guidance Document: Comparative Bioavailability Standards: Formulations Used for Systemic Effects. Guidance document. Ottawa:, Health Products and Food Branch; 2010.

17. Japan National Institute of Health Sciences. Guideline for Bioequivalence Studies of Generic Products. Guidance document. Tokyo:; 2006.

18. Bolton S. Bioequivalence Studies for Levothyroxine. The AAPS Journal. 2005; 7(1): p. E47-E53.

19. Dissanayake S. Assessing the bioequivalence of analogues of endogenous substances ('endogenous drugs'): considerations to optimize study design. British Journal of Clinical Pharmacology. 2010; 69(3): p. 238-244.

20. Haynes R. Thyroid and Antithyroid Drugs. In Gilman A, Rall T, Nies A, Taylor P, editors. Goodman and Gilman's The Pharmacological Basis of Therapeutics. New York: McGraw-Hill, Inc.; 1993. p. 1361-1383.

21. Dong B. Thyroid and Parathyroid Disorders. In Herfindal E, Gourley D, Hart L, editors. Clinical Pharmacy and Therapeutics. Baltimore: Williams \& Wilkins; 1992. p. 267-306.

22. Pangaro L. Physiology of the Thyroid Gland. In Becker K, editor. Principles and Practice of Endocrinology and Metabolism. Philadelphia: J.B. Lippincott Company; 1990.

23. Schindel F. Consideration of endogenous 
backgrounds in pharmacokinetic analyses: a simulation study. Eur J Clin Pharmacol. 2000; 56: p. 685-688.

24. D'Argenio D, Shumitzky A, Wang X. ADAPT 5 User's Guide: Pharmacokinetic/Pharmacodynamic Systems Analysis Software Los Angeles: Biomedical Simulations Resource; 2009.

25. Seligman P, Dahl N, Strobos J, Kimko H, Schleicher $\mathrm{R}$, Jones $\mathrm{M}$, et al. Single-dose pharmacokinetics of sodium ferric gluconate complex in iron-deficient subjects. Pharmacotherapy. 2004; 24(5): p. 574-83.

26. Schuirmann D. A comparison of the two one-sided tests procedure and the power approach for assessing the equivalence of average bioavailability. Journal of pharmacokinetics and biopharmaceutics. 1987; 15(6): p. 657-80.

27. Baribeault D. Sodium ferric gluconate (SFG) in complex with sucrose for IV infusion: bioequivalence of a new generic product with the branded product in healthy volunteers. Current medical research and opinion. 2011; 27(8): p. 16537.

28. Box G. Some theorems on quadratic forms applied in the study of analysis of variance problems, I. Effect of Inequality of Variance in the One-way Classification. Annals of Mathematical Statistics. 1954; 25(2): p. 290-302.

29. Di Stefano JI. Noncompartmental vs. compartmental analysis: some bases for choicev. Am J Physiol. 1982; 243: p. R1-R6.

30. Jusko WJ. Guidelines for Collection and Analysis of Pharmacokinetic Data. In Burton ME, Shaw LM, Schentag JJ, Evans WE, editors. Applied Pharmacokinetics \& Pharmacodynamics: Principles of Therapeutic Drug Monitoring. Baltimore: Lippincott Williams \& Wilkins; 2006. p. 8-29.

31. Van Wyck D. Labile iron: manifestations and clinical implications. Journal of the American Society of Nephrology. 2004; 15(Suppl 2): p. S107-11.

32. Porter J, Rafique R, Srichairatanakool S, Davis B, Shah F, Hair T, et al. Recent insights into interactions of deferoxamine with cellular and plasma iron pools: Implications for clinical use. Annals of the New York Academy of Sciences. 2005; 1054: p. 155-68.

33. Combrink M, McFadyen M, Miller R. A comparison of the standard approach and the NONMEM approach in the estimation of bioavailability in man. The Journal of pharmacy and pharmacology. 1997; 49(7): p. 731-3.

34. Dubois A, Gsteiger S, Balser S, Pigeolet E, Steimer J, Pillai G, et al. Pharmacokinetic similarity of biologics: analysis using nonlinear mixed-effects modeling. Clinical pharmacology and therapeutics.
2012; 91(2): p. 234-42.

35. Dubois A, Gsteiger S, Pigeolet E, Mentre F. Bioequivalence tests based on individual estimates using non-compartmental or model-based analyses: evaluation of estimates of sample means and type I error for different designs. Pharmaceutical research. 2010; 27(1): p. 92-104.

36. Dubois A, Lavielle M, Gsteiger S, Pigeolet E, Mentre F. Model-based analyses of bioequivalence crossover trials using the stochastic approximation expectation maximisation algorithm. Statistics in medicine. 2011; 30(21): p. 2582-600.

37. Fradette C, Lavigne J, Waters D, Ducharme M. The utility of the population approach applied to bioequivalence in patients: comparison of 2 formulations of cyclosporine. Therapeutic drug monitoring. 2005; 27(5): p. 592-600.

38. $\mathrm{Hu} \mathrm{C}$, Moore K, Kim Y, Sale M. Statistical issues in a modeling approach to assessing bioequivalence or PK similarity with presence of sparsely sampled subjects. Journal of pharmacokinetics and pharmacodynamics. 2004; 31(4): p. 321-39.

39. Kaniwa N, Aoyagi N, Ogata H, Ishii M. Application of the NONMEM method to evaluation of the bioavailability of drug products. Journal of pharmaceutical sciences. 1990; 79(12): p. 1116-20.

40. Panhard X, Mentre F. Evaluation by simulation of tests based on non-linear mixed-effects models in pharmacokinetic interaction and bioequivalence cross-over trials. Statistics in medicine. 2005; 24(10): p. 1509-24.

41. Pentikis H, Henderson J, Tran N, Ludden T. Bioequivalence: individual and population compartmental modeling compared to the noncompartmental approach. Pharmaceutical research. 1996; 13(7): p. 1116-21.

42. Davit BM, Connor DP. United States of America. In Kanfer I, Shargel L, editors. Generic Drug Product Development: International Regulatory Requirements for Bioequivalence. New York: Informa Healthcare; 2010. p. 271-272.

43. Haidar SH, Davit D, Chen ML, Connor D, Lee LM, Li QH, et al. Bioequivalence Approaches for Highly Variable Drugs and Drug Products. Pharmaceutical Research. 2008 January; 25(1): p. 237-241. 


\section{ABBREVIATIONS}

\begin{tabular}{|c|c|}
\hline Term & Definition \\
\hline AIC & Akaike information criterion \\
\hline ANOVA & Analysis of variance \\
\hline $\mathrm{AUC}_{\text {pred }}$ & $\begin{array}{l}\text { Area under the serum-time curve of SFGC-I, from the beginning of the infusion to } \\
\text { infinity, calculated as dose divided by CL }\end{array}$ \\
\hline $\mathrm{AUC}_{0-36}$ & Area-under-the-concentration-time-curve from time zero to 36 hours \\
\hline BA & Bioavailability \\
\hline $\mathrm{BE}$ & Bioequivalence \\
\hline BIC & Bayesian information criterion \\
\hline BLQ & Below the limit of quantitation \\
\hline $\mathrm{CI}(\mathrm{s})$ & Confidence interval(s) \\
\hline $\mathrm{CL}_{1}$ & Clearance of SFGC-I to the RES \\
\hline $\mathrm{CL}_{2}$ & Clearance of SFGC-I directly to transferrin \\
\hline $\mathrm{CL}_{3}$ & Clearance of iron entering and exiting the marrow and red blood cell compartment \\
\hline $\mathrm{CL}_{4}$ & Clearance of TBI to the RES \\
\hline $\mathrm{CL}$ & Total clearance for SFGC-I, calculated as the sum of $\mathrm{CL}_{1}+\mathrm{CL}_{2}$ \\
\hline Cmax $_{\text {pred }}$ & $\begin{array}{l}\text { Maximum predicted serum concentration of serum SFGC-I over the 72-hour sampling } \\
\text { period }\end{array}$ \\
\hline C_NTBI_P1 & Concentration of non-transferrin-bound iron during Period 1 \\
\hline C_NTBI_P2 & Concentration of non-transferrin-bound iron during Period 2 \\
\hline FDA & Food and Drug Administration \\
\hline Frel & Relative bioavailability \\
\hline ITS & Iterative two-stage \\
\hline $\mathrm{Km}$ & $\begin{array}{l}\text { Iron concentration associated with half of the maximal rate of exchange between the RES } \\
\text { and TBI compartments }\end{array}$ \\
\hline LSM & Least squares mean \\
\hline MAP & Maximum a posteriori \\
\hline NTBI & Non-transferrin bound iron \\
\hline OGD & Office of Generic Drugs \\
\hline PK & Pharmacokinetics \\
\hline PPK & Population pharmacokinetics \\
\hline $\mathrm{RBC}$ & Red blood cells \\
\hline RES & Reticuloendothelial system \\
\hline SFGC & Sodium ferric gluconate complex \\
\hline SFCG-I & Iron bound to sodium ferric gluconate complex \\
\hline STS & Standard two-stage \\
\hline $\mathrm{T}_{1 / 2}$ & Apparent first-order terminal elimination half-life of SFGC-I \\
\hline TBI & Transferrin-bound iron \\
\hline TI & Total iron \\
\hline Vmax & Maximal rate of exchange between the RES and TBI compartments \\
\hline $\mathrm{V} \mathrm{RBC}$ & Marrow and red blood cell compartment \\
\hline V_RES & Volume of distribution associated with the RES \\
\hline Vss & Apparent steady-state volume of distribution of SFGC-I \\
\hline V_TBI & Volume of distribution associated with TBI \\
\hline
\end{tabular}


Table 5. Summary of the Statistical Analyses for Ln-transformed PK Parameters

\begin{tabular}{|c|c|c|c|c|c|c|c|c|c|}
\hline \multirow[t]{2}{*}{ Study } & \multirow[t]{2}{*}{ Analysis Type } & \multirow[t]{2}{*}{ Analyte } & \multicolumn{3}{|c|}{$\mathrm{Cmax}(\mathrm{mcg} / \mathrm{dL})$} & \multicolumn{3}{|c|}{$\mathrm{AUC}(\mathrm{mcg} \cdot \mathrm{h} / \mathrm{dL})^{\mathrm{d}}$} & \multirow{2}{*}{$\begin{array}{l}\text { Statistica } \\
\text { Power to } \\
\text { Prove BE }\end{array}$} \\
\hline & & & Test $^{\mathrm{a}}$ & Reference $^{a}$ & Ratio $^{b}(90 \%$ CI $)$ & Test $^{\mathrm{a}}$ & Reference $^{a}$ & $\begin{array}{l}\text { Ratio }^{\mathrm{b}}(90 \% \\
\text { CI })\end{array}$ & \\
\hline \multirow[t]{3}{*}{$\begin{array}{l}\text { Study } 1 \\
(n=29)\end{array}$} & Compartmental & SFGC-I & 1127 & 1256 & $89.9(85.9-94.0)$ & 2534 & 2828 & $\begin{array}{l}89.7(85.7 \text { - } \\
93.9)\end{array}$ & $>80 \%$ \\
\hline & Noncompartmental $^{c}$ & TI & 1338 & 1279 & $104.6(86.3-127.0)$ & 8807 & 9071 & $\begin{array}{l}97.1(74.1- \\
127.2)\end{array}$ & $<40 \%$ \\
\hline & & TBI & 171.1 & 178.4 & $95.9(83.4-110.2)$ & 3364 & 2811 & $\begin{array}{l}119.7(20.5 \\
-698.6)\end{array}$ & \\
\hline \multirow[t]{2}{*}{$\begin{array}{l}\text { Study } 2 \\
(n=240)\end{array}$} & Noncompartmental $^{c}$ & TI & 3106 & 3098 & $100.4(96.5-104.5)$ & 11101 & 11033 & $\begin{array}{l}99.7(94.2- \\
105.5)\end{array}$ & $>80 \%$ \\
\hline & & TBI & NA & NA & $86.8(82.7-91.1)$ & NA & NA & $\begin{array}{l}92.4(85.6- \\
99.7)\end{array}$ & \\
\hline
\end{tabular}

NA - Information not available in publication

${ }^{a}$ Geometric mean for Study 1 and arithmetic mean for Study 2 (geometric mean not reported for Study 2)

${ }^{b}$ Ratio of geometric means (Test/Reference)

${ }^{\mathrm{c}}$ Baseline-adjusted $\mathrm{PK}$ parameters

${ }^{\mathrm{d}} \mathrm{AUC}_{\text {inf }}$ for Study 1 and $\mathrm{AUC}_{0-36}$ for Study 2 
J Pharm Pharm Sci (www.cspsCanada.org) 16(3) 424 - 440, 2013 